\title{
Postsynaptic cleft density changes with combined exercise protocols in an experi- mental model of muscular hypertrophy
}

\author{
Jurandyr Pimentel Neto, Lara Caetano Rocha, Carolina dos Santos Jacob, Gabriela Klein Barbosa, Adriano \\ Polican Ciena
}

Laboratory of Morphology and Physical Activity (LAMAF), Institute of Biosciences (IB), São Paulo State University

(UNESP), Rio Claro-SP, Brazil

\begin{abstract}
The vertical ladder-based protocols contribute to the NMJ junction's adaptations, and when combined with and without load, can be potentiated. The present study aimed to investigate postsynaptic regions of the biceps brachii muscle in adult male Wistar rats submitted to different vertical ladder-based protocols (Sedentary - S; Climbing - C; Climbing with Load - LC and Combined Climbing - CC). The protocols (C, LC, CC) were performed in 24 sessions, $3 \mathrm{x} /$ week, for 8 weeks. The myofibrillar ATPase analysis showed an increase in crosssectional area (CSA) of the muscle fibers Type I in all trained Groups; Type II in C and LC and reduction in CC; Type IIx higher in all trained Groups. In the postsynaptic cleft, the stained area presents smaller in Groups $\mathrm{C}, \mathrm{LC}$, and CC; the total area showed smaller than LC and higher in C and CC. The stained and total perimeter, and dispersion showed a reduction in $\mathrm{C}, \mathrm{LC}$, and $\mathrm{CC}$, higher maximum diameter in Groups $\mathrm{C}$ and $\mathrm{CC}$, and decreased in LC. Regarding the postsynaptic cleft distribution, the stained area presented a decrease in all trained Groups. The integrated density presented higher principally in CC. The NMJ count showed an increase in all trained Groups. We concluded that the vertical ladder-based protocols combined contributed to the postsynaptic region adaptations.
\end{abstract}

Key words: neuromuscular junction, muscle fiber type, synapse, hypertrophy, morphometry, immunohistochemistry.

Correspondence: Prof. Dr. Adriano Polican Ciena, Institute of Biosciences (IB), Laboratory of Morphology and Physical Activity (LAMAF), São Paulo State University (UNESP), Avenue 24A 1515, Bela Vista, 13506-900 for 13506-900. Rio Claro-SP, Brazil. Tel. +55.19.3526-4346. E-mail: adriano.ciena@unesp.br

Contributions: JPN, APC, conceived and designed the research study, prepared the figures and wrote the paper; JPN, LCR, GKB, CSJ, contributed to protocol; JPN, LCR, GKB, CSJ, APC, contributed to the analysis and interpretation of data. All authors have read and approved the final version of the manuscript.

Conflict of interest: The authors declare no conflicts of interests.

Funding: The work was supported by Grants \#2017/12525-1 and \#2017/21977-3, São Paulo Research Foundation (FAPESP), in part by the Coordenação de Aperfeiçoamento de Pessoal de Nível Superior-Brasil (CAPES) - Finance Code 001 and Edital PROPG 05/2021.

Ethical approval: Committee on Ethics in Animal Use of the Institute of Biosciences of the São Paulo State University (CEUA - n. 2207).

Availability of data and material: All relevant data are within the paper. 


\section{Introduction}

The neuromuscular junction (NMJ) forms a synaptic connection, and its structure consists of three components: axonal terminal, muscle fibers, and Schwann cells. ${ }^{1,2}$ Its morphological changes are commonly recognized by immunostaining ( $\alpha$-bungarotoxin) that in a way effective represent the synaptic function and is associated with pathologies such as amyotrophic lateral sclerosis (ALS), spinal muscular atrophy (SMA), Duchenne muscular dystrophy (DMD), and with physical exercise protocols like swimming and vertical ladder-based resistance training. ${ }^{3-7}$

Skeletal muscle fibers in humans are characterized by their contraction speed or metabolic speed, divided into three main types: type I (slow oxidative), type II (oxidative glycolytic), and type IIb (fast glycolytic); Meanwhile, fibers IIb in rats are classified as IIx. ${ }^{7,8}$ The muscle contraction cycle requires energy generated by adenosine triphosphate (ATP) nucleotides generated by aerobiosis in the mitochondria, particularly abundant in adult muscle fibers. The contraction is stimulated through motor neurons, connected to the activated muscle fibers through the NMJ.9,10

The acetylcholine released in the synaptic cleft interacts with specific receptors (AChR) and generates an action potential across the surface of the muscle cell, which diffuses rapidly within muscle tissue through a network of specialized tubules called $\mathrm{T}$ tubules. ${ }^{11,12}$ The action potential activates the dihydropyridine receptor opening that allows the influx of calcium $\left(\mathrm{Ca}^{2+}\right)$ into the cytoplasm and stimulates the ryanodine receptors to release more $\mathrm{Ca}^{2+}$ from the sarcoplasmic reticulum, in turn, triggers the interaction between contractile proteins and causes a shortening of muscle fiber. ${ }^{13}$

NMJ has wide strands regarding its plasticity in the face of stimuli such as aging (sarcopenia), use of pharmacological drugs, and physical exercise due to the direct relationship with the skeletal striated muscle, this correlation with the peripheral nervous system shows an adaptation of the muscle fibers to the pre and postsynaptic activity of NMJ., 5 -14-16

In recent studies, adaptations in size and perimeter of the postsynaptic region have been unleashed by exercise protocols in adult and elderly rats, evidencing their tissue plasticity. ${ }^{7,17}$ Some studies have shown resistance training promotes the increase in the perimeter and the area of the terminal nerve branch, in the area occupied by the vesicles and AChR, as well as in the dispersion of both presynaptic acetylcholine vesicles and postsynaptic AChR. ${ }^{5,15}$ Vertical ladder-based protocol with overload in an experimental model has been used in research and until nowadays. This protocol mainly assesses functional aspects, such as increased strength, and morphological aspects of muscle tissue, specifically the sarcomeres, ${ }^{18}$ the peripheral nervous system, ${ }^{19}$ and the myotendinous junction. ${ }^{20}$ In addition, it can be applied to elderly animals, ${ }^{21}$ but it is mainly used for adult animals, and has shown positive results such as tissue maintenance, hypertrophy, and increased strength. ${ }^{22}$

The biceps brachii muscle is constantly used in daily activities and works in different physical exercise protocols with and without load. The combined vertical ladder-based protocols can demonstrate new benefits to the biceps brachii tissue morphology and in their postsynaptic elements. Furthermore, the specific development of the different fiber types involved in executing each regular training can be associated with the postsynaptic cleft structural adaptations and density growth. The present study aimed to investigate the vertical ladder-based protocols adaptations in the postsynaptic region in adult rats.

\section{Materials and Methods}

\section{Animals}

Thirty-two male Wistar rats 90-days-old were divided into 4 Groups $(\mathrm{n}=8)$ : Sedentary (S) - was not submitted to any experimental protocol; Climbing (C) - performed climbs with bodyweight; Climbing with load (LC) - performed climbs with additional progressive load; Combined Climbing (CC) - performed combined (training sessions alternated with and without load) climbing protocols. During the experimental period, the animals were allocated in cages $(33 \times 40 \times 16 \mathrm{~cm}, \mathrm{n}=4)$, under the conditions of temperature monitoring $\left(23 \pm 2^{\circ} \mathrm{C}\right)$ and $12 \mathrm{~h}$ light/dark period, with food and water ad libitum. The procedures applied in this study were approved by the Committee on Ethics in Animal Use of the Institute of Biosciences of the São Paulo State University (CEUA - n 2207).

\section{Climbing protocol (C)}

The $\mathrm{C}$ group performed an 8 -week training protocol (3x/week) with 24 sessions using a vertical ladder $\left(110 \times 18 \mathrm{~cm}, 2 \mathrm{~cm}\right.$ grid, $80^{\circ}$ incline). The sessions consisted of 9 climbs without additional load to the top of the ladder, where the rats were allowed to rest for 60 seconds in each. ${ }^{18,20}$

\section{Climbing with load protocol (LC)}

The LC group performed an 8-week resistance training protocol $(3 \mathrm{x} /$ week $)$ with a total of 24 sessions using a vertical ladder ( $110 \times 18 \mathrm{~cm}, 2 \mathrm{~cm}$ grid, $80^{\circ}$ incline). The sessions consisted of 4 to 9 climbs with additional and progressive load to the top of the ladder, where the rats were allowed to rest for $120 \mathrm{~s}$ in each. ${ }^{18}$

The $1^{\text {st }}, 2^{\text {nd }}, 3^{\text {rd }}$ and $4^{\text {th }}$ climbs were performed respectively with $50 \%, 75 \%, 90 \%$, and $100 \%$ of animal body mass, and in the subsequent climbs, a $30 \mathrm{~g}$ of extra progressive load was added until the $9^{\text {th }}$ climb completed or exhaustion based on the protocol of Hornberger and Farrar. ${ }^{18,20,23}$ The load was fixed to the proximal region of the tail.

\section{Combined climbing protocol (CC)}

The CC protocol consists of 8 -week training ( $3 \mathrm{x} /$ week) using a vertical ladder $\left(110 \times 18 \mathrm{~cm}, 2 \mathrm{~cm}\right.$ grid, $80^{\circ}$ incline $)$ with and without an additional load in alternate sessions, for example, in the $1^{\text {st }}$ session of the protocol, the CC group executed 4 to 9 climbs with additional and progressive load to the top of the ladder like the LC group. In the $2^{\text {nd }}$ session, the group completed nine climbs without additional load like the $\mathrm{C}$ group and so on interspersed until the $24^{\text {th }}$ session was completed.

\section{Body and muscle mass}

The animal's body mass was measured with a digital balance (TOMATETM 440) weekly to establish the additional loads in LC and CC Groups. In the last protocol week, we obtained the means \pm SD of group mass of the initial body mass (P1) and final body mass (P2), and we made the statistical analysis with Graph Pad Prism $^{\mathrm{TM}}$ 8.0.1 Software with two-way ANOVA analysis and Bonferroni post-hoc test $(\mathrm{p}<0.05)$.

We measured the biceps brachii muscle mass with a semi-analytical balance (Marte Científica ${ }^{\mathrm{TM}} \mathrm{AD} 330$ ) at the end of experimental protocols. After obtaining the data of means $\pm \mathrm{SD}$, we performed the statistical analysis with Graph Pad Prism ${ }^{\text {TM }}$ 8.0.1 software with one-way ANOVA analysis and a Bonferroni post-hoc test $(\mathrm{p}<0.05)$.

\section{Histochemistry}

The belly samples of biceps brachii muscle $(n=5)$ of experi- 
mental groups were dissected and cryofixed in liquid nitrogen allocated in a cryomold $\left(\right.$ Sakura $^{\circledR}{ }^{\circledR}$ Tissue-Tek ${ }^{\circledR}$ - 4557 Standard $20 \times 25 \times 5 \mathrm{~mm})$. We performed transversal sections (10 $\mu \mathrm{m}$ thick) (Cryostat HM 505 E, MICROM ${ }^{\mathrm{TM}}$ ) and used the histochemical reaction for myosin adenosine triphosphatase (ATP) and the $\mathrm{pH}$ lability to differentiate the fiber types I, II, and IIx. For $\mathrm{pH} 4.3$ and 4.6, the sections were pre-incubated in $0.1 \mathrm{M}$ sodium acetate buffer solution and $10 \mathrm{mM}$ ethylenediaminetetraacetic acid (EDTA) solution for $10 \mathrm{~min}$ at $4^{\circ} \mathrm{C}$. All the sections were incubated in a solution containing ATP $(10 \mathrm{mg})$, glycine/ $\mathrm{NaCl}$ buffer $(10 \mathrm{~mL}), \mathrm{CaCl}_{2}$ (in $\mathrm{pH}$ 9.4), and dithiothreitol for $30 \mathrm{~min}$. The sections were incubated in $2 \%$ cobalt chloride for $7 \mathrm{~min}$ dehydrated in a series of alcohol concentrations finished in xylol. ${ }^{18}$

\section{Muscle fiber types}

The images were obtained by a Zeiss ${ }^{\mathrm{TM}}$ Axioscope light microscope. For the procedure of image acquisition, we use random labeling of the histological slides to photograph and quantify the images with low bias. Subsequently, the morphometry was processed with the Image JTM software applied to slides with ATPase in each $\mathrm{pH}$. We quantified the cross-section area (CSA) of Type I, Type II, and Type IIx myofibers ( $n=100 /$ fiber type/group) in 200X magnification images. Data were analyzed using KruskalWallis with Dunn's post-hoc test $(\mathrm{p}<0.05)$ by GraphPad Prism ${ }^{\mathrm{TM}}$ 8.0.1.

The stereology analysis of numerical density (\%) was performed using the Image JTM software in $200 \mathrm{X}$ magnification images of Type I, II, and IIx muscle fibers ( $\mathrm{n}=10$ images/group); they were measured by counting the number of myofibers per photographed field, and their group means percentage was calculated. ${ }^{7}$

\section{Immunohistochemistry}

The samples of biceps brachii ( $\mathrm{n}=3$ in each) of experimental groups were dissected and cryofixed in liquid nitrogen. We performed longitudinal sections (100 $\mu \mathrm{m}$ thickness) (Cryostat HM $\left.505 \mathrm{E}, \mathrm{MICROM}^{\mathrm{TM}}\right)$. Such sections were collected in silanized slides, pre-treated with $3 \%$ EDTA solution, and washed $(3 \times 5 \mathrm{~min})$ in phosphate-buffered saline (PBS) containing $1 \%$ bovine serum albumin (BSA). After, the sections were incubated overnight at $4^{\circ} \mathrm{C}$ in a solution containing $\alpha$-bungarotoxin conjugated rhodamine (BTX; Molecular Probes ${ }^{\circledR}$, Eugene, OR, USA; T-1175), diluted 1:600 in PBS. The biceps brachii sections were then washed in PBS and BSA before being coated with Prolong (Molecular Probes $^{\circledR}$; P10144), coverslips applied, and stored at $-20^{\circ} \mathrm{C}$ until analysis. $^{7}$

\section{Postsynaptic cleft structure}

For the postsynaptic cleft structure analysis, 20 images were used for each experimental group by an Olympus ${ }^{\mathrm{TM}}$ BX61 Fully Motorized Fluorescence Microscope (Shinjuku, Japan) equipped with a Fluorescence UIS2 optical system by TexasRed filter. The images were obtained using a monochromatic camera Orca-Flash
2.8 (Hamamatsu, Japan), with the CellSens 11 Software (Olympus $^{\mathrm{TM}}$ ) in $1000 \mathrm{X}$ magnification.

After, we measured with Image ${ }^{\mathrm{TM}}$ software the postsynaptic cleft structures variables: stained postsynaptic cleft area $\left(\mu \mathrm{m}^{2}\right)$, total postsynaptic cleft area $\left(\mu \mathrm{m}^{2}\right)$, stained postsynaptic cleft perimeter $(\mu \mathrm{m})$, total postsynaptic cleft perimeter $(\mu \mathrm{m})$, the postsynaptic cleft dispersion (\%) regarding the variables of area relation, and the postsynaptic cleft maximum diameter $(\mu \mathrm{m})$ to determine the higher distance between their limits. ${ }^{21}$ Data were analyzed using Kruskal-Wallis with Dunn's post-hoc test $(\mathrm{p}<0.05)$ by GraphPad Prism ${ }^{\mathrm{TM}}$ 8.0.1.

\section{Postsynaptic cleft region}

About the postsynaptic cleft region $(n=20)$, we selected 400x magnification images to evaluate the distribution and density. The stained area $\left(\mu \mathrm{m}^{2}\right)$ of the postsynaptic cleft region was binarized and measured, establishing the means of individual postsynaptic clefts area per image. The NMJ Count (unit) of the postsynaptic cleft means were determined with a point-by-point numerical count per image. Integrated density (PPI), which is the sum of all the pixel intensities in the ROI, is a methodology for regular 2D analysis (analyze/ set measurements/ select integrated density). Image ${ }^{\mathrm{TM}}$ reports the integrated density, which is the product of area and mean intensity. Data were analyzed using Kruskal-Wallis with Dunn's post-hoc test $(\mathrm{p}<0.05)$ by GraphPad Prism ${ }^{\mathrm{TM}} 8.0 .1$ (Figure 1).

\section{Results}

\section{Body and muscle mass}

In the $\mathrm{S}$ group, the body mass difference between P1 and P2 was $15.3 \%(p<0.001)$. The biceps brachii muscle mass in $\mathrm{S}$ group was higher than $\mathrm{C}$ group and smaller than LC and CC groups (Table 1). In the $\mathrm{C}$ group, the body mass difference between $\mathrm{P} 1$ and $\mathrm{P} 2$ was $18.9 \%(\mathrm{p}<0.0001)$. Also, the $\mathrm{C}$ group presents a smaller muscle mass than the other experimental groups (Table 1). In the LC group, the body mass difference between P1 and P2 was $20.7 \%$ $(\mathrm{p}<0.0001)$. The LC group showed the highest muscle mass in relation to the $\mathrm{S}$ group $(\mathrm{p}<0.001)$, to $\mathrm{C}$ group $(\mathrm{p}<0.0001)$, and with $\mathrm{CC}$ group (Table 1). In the $\mathrm{CC}$ group, the body mass difference between P1 and P2 was $17.5 \%(\mathrm{p}<0.0001)$. The $\mathrm{CC}$ group showed higher muscle mass than $\mathrm{S}$ and $\mathrm{C}$ group and smaller muscle mass than LC $(\mathrm{p}<0.05)$ (Table 1).

\section{Fiber type cross-section area}

The CSA of type I, II, and IIx from all experimental groups' biceps brachii muscle was quantified (Table 2). The CSA of type I fibers of the $S$ group was smaller than that found in $C(p<0.0001)$, LC $(\mathrm{p}<0.005)$, and CC. In the C group, the CSA of type I fibers

Table 1. Mean \pm standard deviation of corporal mass (g) and biceps brachii muscle mass (g) in the Sedentary (S), Climbing (C), Climbing with Load (LC), and Combined Climbing (CC) groups. Data was treated statistically through the Kruskal-Wallis analysis of variance, with Dunn's post-hoc test and significance level of $\mathbf{p}<0.05$.

\begin{tabular}{lccccc} 
& & & Groups & LC \\
Body mass (g) & P1 & S & C & $395.5 \pm 29$ & $396 \pm 24.3$ \\
& P2 & $366 \pm 22$ & $395 \pm 29.3$ & $477.5 \pm 31.9^{\#}$ & $465.5 \pm 42.9^{\#}$ \\
\hline Muscle mass (g) & & $422 \pm 35^{*}$ & $470 \pm 37.1^{\#}$ & $0.281 \pm 0.044^{* \#}$ \\
\hline
\end{tabular}

Body mass: *SP1 $\neq \mathrm{SP} 2(\mathrm{p}<0.001) ;{ }^{*} \mathrm{CP} 1 \neq \mathrm{CP} 2, \mathrm{LCP} 1 \neq \mathrm{LCP} 2, \mathrm{CCP} 1 \neq \mathrm{CCP} 2(\mathrm{p}<0.0001) ;$ muscle mass: *S $\neq \mathrm{LC}(\mathrm{p}<0.001) ; \# \mathrm{C} \neq \mathrm{LC}(\mathrm{p}<0.0001) ; \S \mathrm{LC} \neq \mathrm{CC}(\mathrm{p}<0.05)$. 
was larger than LC $(p<0.0001)$ and CC $(p<0.0001)$. Furthermore, the CSA of type I fibers of the LC group was larger compared to CC (Table 2). The CSA of type II fibers of the $\mathrm{S}$ group was smaller than that found in $\mathrm{C}$ and $\mathrm{LC}(\mathrm{p}<0.01)$; however, it was larger than CC. The CSA of type II fibers of the $\mathrm{C}$ group was larger than LC $(p<0.0001)$ and CC. The CSA of type II fibers of the LC group was larger compared to $\mathrm{CC}(\mathrm{p}<0.001)$ (Table 2$)$. Additionally, the CSA of type IIx of $\mathrm{S}$ group was smaller than $\mathrm{C}(\mathrm{p}<0.0001)$, LC $(p<0.001)$, and $C C(p<0.0001)$. In the $C$ group, the CSA of type IIx fibers was larger than LC $(\mathrm{p}<0.0001)$ and CC. The CSA of type IIx of LC group was smaller than CC $(\mathrm{p}<0.0001)$ (Table 2$)$.

\section{Postsynaptic cleft morphometry}

The stained area of the $\mathrm{S}$ group was larger compared to C, LC, and $\mathrm{CC}$. The $\mathrm{C}$ group presented the stained area larger than $\mathrm{LC}$ and CC. Besides, the stained area of the LC group was smaller compared to $\mathrm{CC}$ (Figure 2I). The $\mathrm{S}$ group's total area was smaller than $\mathrm{C}$ and $\mathrm{CC}$; however, it was larger compared to $\mathrm{LC}$. The total area of the $\mathrm{C}$ group was larger than LC and smaller than CC. Furthermore, the total area of the LC group was smaller compared to $\mathrm{CC}$ (Figure 2I). The stained perimeter of the $\mathrm{S}$ group was larger than $\mathrm{C}, \mathrm{LC}$, and $\mathrm{CC}$. The stained perimeter of the $\mathrm{C}$ group presented larger compared to LC and CC. In the LC group, the stained perimeter was smaller than CC (Figure 2II). The total perimeter of the $\mathrm{S}$ group was larger than $\mathrm{C}, \mathrm{LC}$, and $\mathrm{CC}$. Besides, the total perimeter of the $\mathrm{C}$ group was larger compared to $\mathrm{LC}$ and $\mathrm{CC}$. In the LC group, the total perimeter was smaller than CC (Figure 2II). The endplate dispersion of the $\mathrm{S}$ group was larger compared to $\mathrm{C}$, LC $(p<0.05)$, and CC $(p<0.005)$. The endplate dispersion of the $C$ group was larger than LC and CC. Furthermore, the group LC presented an endplate dispersion larger than CC (Figure 2III).

Additionally, the maximum cleft diameter of the $\mathrm{S}$ group was smaller compared to $\mathrm{C}$ and CC; however, it was larger than LC. The maximum cleft diameter of the $\mathrm{C}$ group was larger than $\mathrm{LC}$ and smaller compared to CC (Figure 2IV).

\section{Postsynaptic cleft distribution}

The stained area data of the S group's postsynaptic distribution was larger compared to C, LC, and CC. Besides, the stained area data of the $\mathrm{C}$ group's postsynaptic distribution was larger than LC and smaller than CC. In the LC group, the postsynaptic distribution's stained area data was smaller than that found in CC (Figure

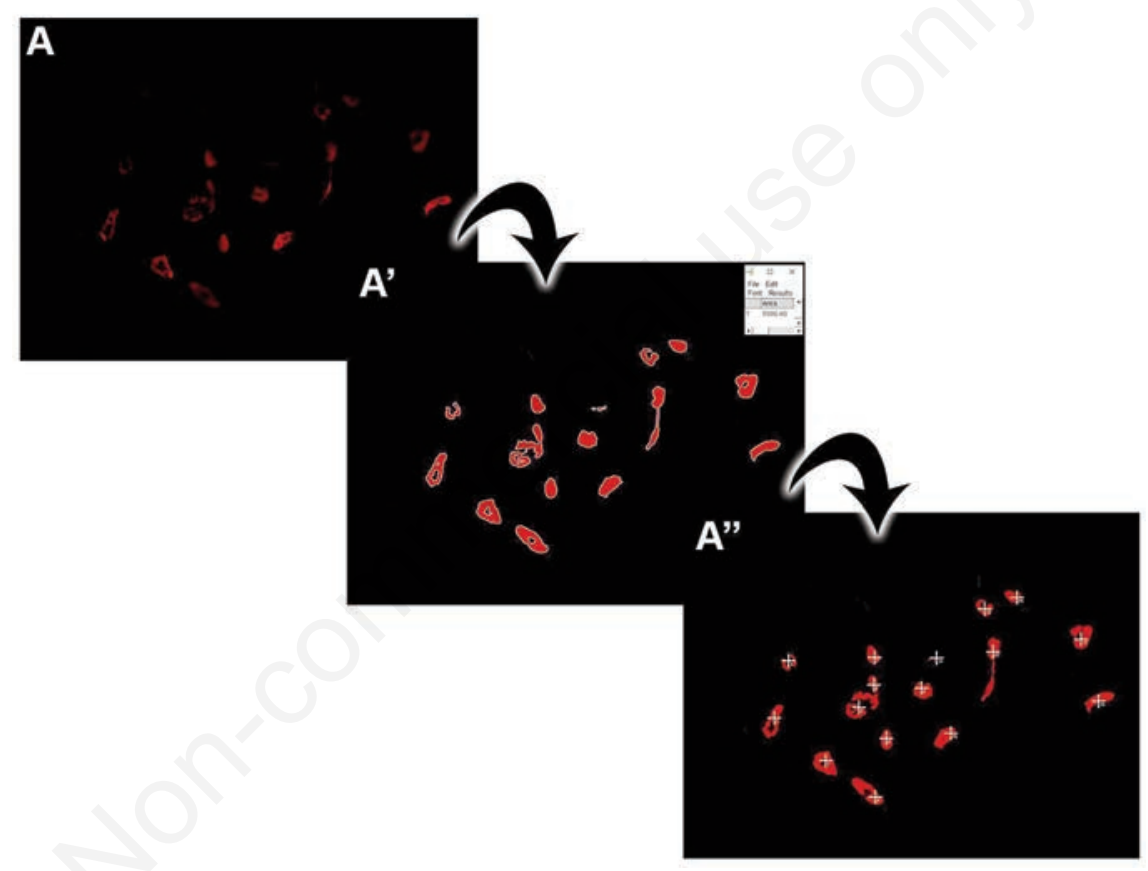

Figure 1. Postsynaptic region image and methodology example to measure the integrated density (A). A') stained area with threshold configuration; A") multi points used for NMJ count. Magnification: 400x.

Table 2. Mean \pm standard deviation of cross-section area $\left(\mu \mathrm{m}^{2}\right)$ and mean of the numerical density (\%) of myofibers I, II, IIx in the biceps brachii of the Sedentary (S), Climbing (C), Climbing with Load (LC), and Combined Climbing (CC) groups. Data of CSA fiber type and Numerical Density was treated statistically through the Kruskal-Wallis with Dunn's post-hoc test and significance level of $\mathbf{p}<0.05$.

\begin{tabular}{|c|c|c|c|c|c|}
\hline & & & & & \\
\hline & & S & C & LC & CC \\
\hline $\mathrm{CSA}\left(\mu \mathrm{m}^{2}\right)$ & Type I & $1611.6 \pm 360.4$ & $2802.8 \pm 624.1^{*}$ & $1868.1 \pm 479.5^{\text {*\# }}$ & $1749.2 \pm 437.7^{*}$ \\
\hline & Type II & $2257.9 \pm 625$ & $2418.9 \pm 493.7$ & $2289.5 \pm 587.7$ & $1969.9 \pm 562.9 * \S \sharp$ \\
\hline & Type IIx & $1915.6 \pm 311.3$ & $2747.4 \pm 708.1^{*}$ & $2160.8 \pm 475.9 * \S$ & $2597.4 \pm 567.7^{*}$ \\
\hline Numerical density percentage & Type I & 62.4 & 47.5 & 55.4 & 18.5 \\
\hline & Type II & 29.9 & 27.6 & 27.3 & 32.9 \\
\hline & Type IIx & 7.7 & 24.9 & 17.3 & 48.6 \\
\hline
\end{tabular}

CSA: Type I $* \mathrm{~S} \neq \mathrm{C}, \mathrm{C} \neq \mathrm{LC}, \mathrm{C} \neq \mathrm{CC}(\mathrm{p}<0.0001), \# \mathrm{~S} \neq \mathrm{LC}(\mathrm{p}<0.01)$; Type II * $\mathrm{C} \neq \mathrm{CC}(\mathrm{p}<0.0001), \S \mathrm{LC} \neq \mathrm{CC}(\mathrm{p}<0.001), \S \mathrm{S} \neq \mathrm{CC}(\mathrm{p}<0.01) ;$ Type IIx $* \mathrm{~S} \neq \mathrm{C}, \mathrm{S} \neq \mathrm{CC}, \mathrm{C} \neq \mathrm{LC}, \mathrm{LC} \neq \mathrm{CC}(\mathrm{p}<0.0001) ; \S \mathrm{S} \neq \mathrm{LC}$ $(\mathrm{p}<0.001)$. 
3I). The NMJ count per image of the $\mathrm{S}$ group presented larger than $\mathrm{C}$ and LC; however, it was smaller than the CC group. The NMJ count of the $\mathrm{C}$ group has a smaller difference than the $\mathrm{LC}$ and $\mathrm{CC}$ group. Besides, the LC Group presented the NMJ count smaller than CC (Figure 3II). The integrated density of S group was smaller than $C, L C$, and significantly smaller than $C C(p<0.0005)$. In the $\mathrm{C}$ group, the integrated density was larger than LC. The LC was significantly smaller than $\mathrm{CC}(\mathrm{p}<0.05)$. Furthermore, the CC integrated density was the highest among all groups (Figure 3III).

\section{Discussion}

The present study demonstrated the postsynaptic region's morphological adaptations and changes in different types of muscle fibers principally with the combined vertical ladder-based protocol's benefits (Table 3). The CSA of muscle fibers showed an increase in type I fibers in the $\mathrm{C}$ group, possibly due to the characteristic oxidative predominance in the biceps brachii muscle of
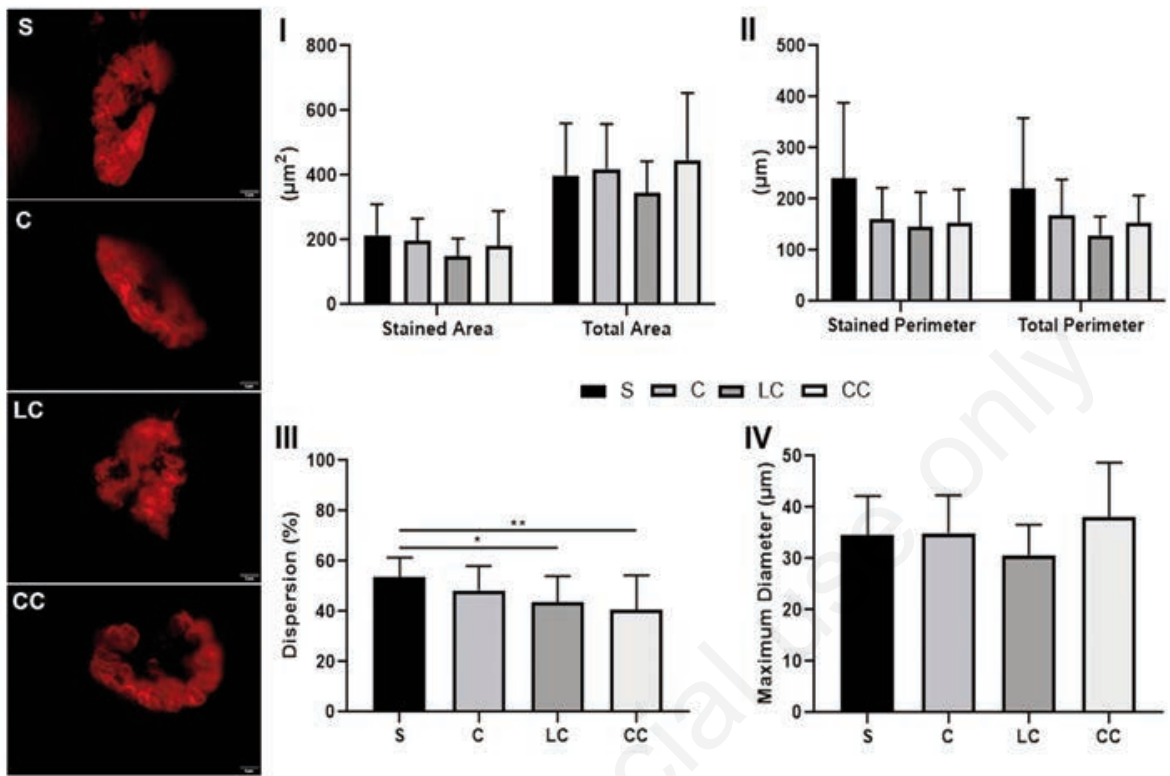

Figure 2. Immunohistochemistry images of the Sedentary group (S), Climbing group (C), Climbing with Load group (LC), and Combined Climbing group (CC). Postsynaptic structure results with means and standard deviations of the data for (I) stained area and total area, (II) stained perimeter and total perimeter, (III) dispersion, and (IV) maximum diameter. Data treated statistically through Kruskal-Wallis with Dunn's post-hoc test and a significance level of $p<0.05 .{ }^{*} S \neq \operatorname{LC}(p<0.05) ;{ }^{* *} \mathbf{S} \neq \mathbf{C C}(\mathrm{p}<0.001)$. Scale bar: $5 \mu \mathrm{m}$.
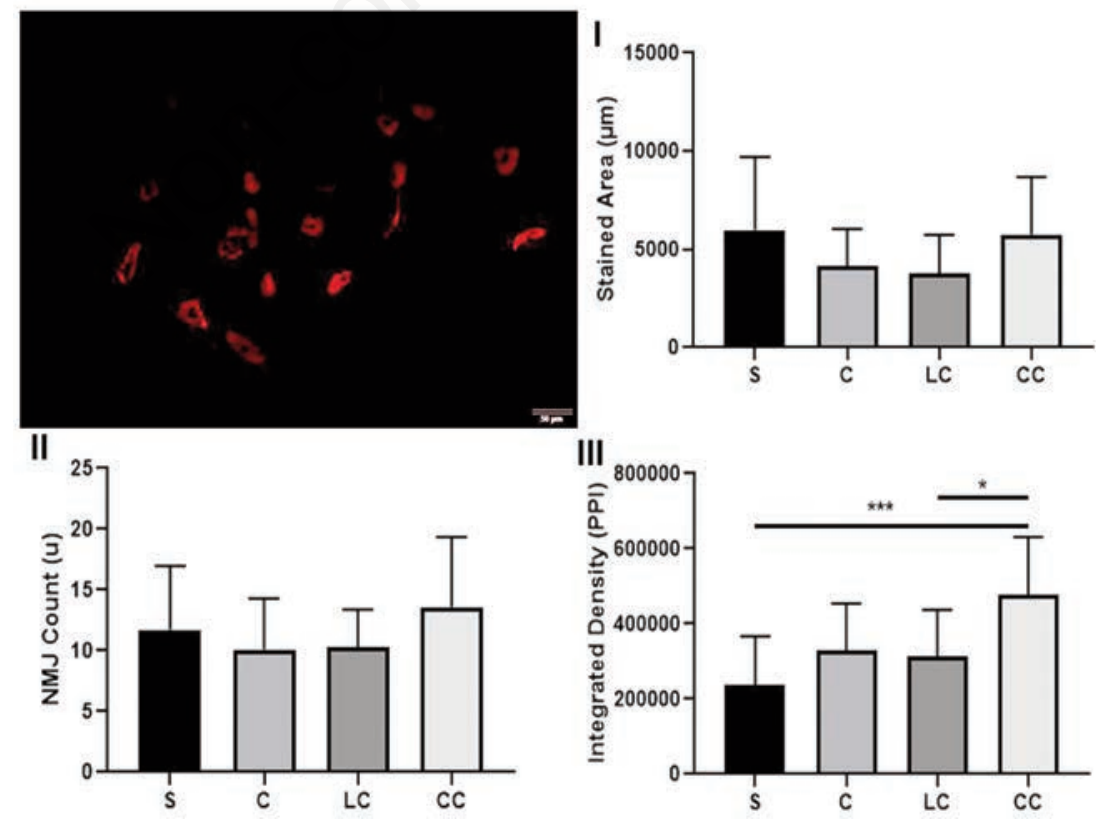

Figure 3. Post-synaptic distribution with means and standard deviations of the (I) stained area, (II) neuromuscular junction (NMJ) count, and (III) integrated density. Data treated statistically through Kruskal-Wallis analysis of variance, with Dunn's post-hoc test and a significance level of $p<0.05 .{ }^{* * *} \mathrm{~S} \neq \mathrm{CC}(\mathrm{p}<0.0005) ;{ }^{*} \mathrm{LC} \neq \mathrm{CC}(\mathrm{p}<0.05)$. Scale bar: $50 \mu \mathrm{m}$. 
rats $^{24}$ and the capacity required by the specific types of muscle fibers to execute the protocol without additional load. ${ }^{25,26}$ Type II and IIx fibers increased in the LC group due to the additional load used to carry out the vertical ladder protocols and the standards established during the protocol sessions, which refer to an experimental model of muscle hypertrophy. ${ }^{22,23}$ While with the vertical ladder-based training in the LC group, we observed greater type II and IIx fibers, indicating hypertrophy of these muscle fibers due to their characteristic of greater strength production, contraction speed, calcium influx, and adaptive sensitivity. ${ }^{27,28}$

In the CC group, the increase in the IIx fibers' CSA demonstrated the adaptation to the glycolytic/oxidative characteristics predominant physiologically and necessary to perform the exercise with and without a load in different sessions. ${ }^{29-31} \mathrm{CSA}$ results present an increase proportional to the fiber density in all experimental groups, demonstrating hypertrophy and hyperplasia of the different fiber types, referring to the physiological requirement/specifical of each proposed exercise protocol. ${ }^{18}$ The changes observed in this study can be defined mainly by the required functionality and the training pattern established for each group, an adaptation similar to what occurs in humans ${ }^{29,32}$ and mainly due to the execution movement required by this muscle before the protocols. ${ }^{31}$

In the LC group, there was a reduction in the postsynaptic cleft structure and of the density in the muscle fibers, possibly due to the functional characteristic of fiber types II and IIx in the action of the ladder-based protocol, which indicates the maintenance of the active zones that adapt and contribute with neurotransmission reducing the access distance from the synaptic vesicles to the $\mathrm{Ca}^{2+}$ channels. ${ }^{33,34}$ The LC group morphometric adaptations are directly related to vertical ladder-based exercise and the muscle's physiological characteristics, as occurred in similar recent experiments, ${ }^{17,18}$ and about the muscle mass association in an experimen- tal model of muscle hypertrophy. ${ }^{19}$ The smaller dispersion in the CC group was directly associated with reducing CSA type I muscle fibers and the predominance with this fiber type in the biceps brachii muscle..$^{22,35}$ Also, the measurements of the postsynaptic cleft in $\mathrm{C}$ group showed morphometric characteristics data with minimal reduction with an increase in density in response to different physical exercises; regarding the LC group, there was mainly a reduction in their structure and density, different from the $\mathrm{CC}$ group, which presented an increase in these aspects due to specific exercises. ${ }^{36,37}$ The changes indicated that the association of the protocols promotes adaptations in adult rats related to the NMJ density, decreasing with the arrival of adulthood, and the $\mathrm{CC}$ protocol maintained their distribution as the main adaptation. ${ }^{37,38}$ The combined exercise protocol can contribute to the understanding of studies in athletes who seek adaptations at the molecular level in the face of different forms, with emphasis on the adaptations of different muscle fiber types, as has already been proven in humans. ${ }^{39,40}$

An elderly contribution with adaptations to peripheral nerves in an experimental model with overload has already been proven; ${ }^{19}$ in this way, a combination of exercises can help maintain postsynaptic clefts. Furthermore, it can contribute to an investigative investigation of different cognitive neurodegenerative pathologies such as Alzheimer, ${ }^{41}$ Parkinson, ${ }^{42}$ and epilepsy, ${ }^{43}$ but molecular analyzes will be necessary to prove these benefits.

We concluded the combined vertical ladder-based protocol demonstrated an increase in the biceps brachii muscle mass, indicating hypertrophy. Enabling the necessary action for the muscle fibers, mainly type IIx, which had to increase fiber density probably associated with the increase of postsynaptic clefts density. In the NMJ occurs an increase in the total area and the maximum diameter of the postsynaptic cleft structure and reduced dispersion.

Table 3. Quantitative results obtained in all groups that performed the vertical ladder-based protocols classified by + (reasonable increase); ++ (considerable increase); +++ (expressive increase); - (reasonable reduction); -- (considerable reduction); --- (expressive reduction).

\begin{tabular}{|c|c|c|c|c|}
\hline & & & der-bs & \\
\hline & & C & LC & $\mathrm{CC}$ \\
\hline Mass & Body mass (g) & ++ & +++ & + \\
\hline & Muscle mass (mg) & - & ++ & + \\
\hline Fiber type & CSA type I $\left(\mu m^{2}\right)$ & +++ & ++ & + \\
\hline & CSA type II $\left(\mu m^{2}\right)$ & ++ & + & - \\
\hline & CSA type IIx $\left(\mu m^{2}\right)$ & +++ & + & ++ \\
\hline & Density type I (\%) & -- & - & --- \\
\hline & Density type II (\%) & -- & - & + \\
\hline & Density type IIx (\%) & ++ & + & +++ \\
\hline Postsynaptic structure & Stained area $\left(\mu m^{2}\right)$ & - & --- & -- \\
\hline & Total area $\left(\mu \mathrm{m}^{2}\right)$ & + & - & ++ \\
\hline & Stained perimeter $(\mu \mathrm{m})$ & - & --- & -- \\
\hline & Total perimeter $(\mu \mathrm{m})$ & - & --- & -- \\
\hline & Dispersion (\%) & - & -- & --- \\
\hline & Maximum diameter $(\mu \mathrm{m})$ & + & - & ++ \\
\hline & Stained area $\left(\mu m^{2}\right)$ & -- & --- & - \\
\hline Postsynaptic distribution & NMJ count (un) & -- & - & + \\
\hline & Integrated density (PPI) & ++ & + & +++ \\
\hline
\end{tabular}




\section{Acknowledgements}

We thank Prof. Dr. Henrique Ferreira from the Department of Biochemistry and Microbiology and his students from the Bacteria Genetics Laboratory for providing the Fluorescence Microscope.

\section{References}

1. Darabid H, Perez-Gonzalez AP, Robitaille R. Neuromuscular synaptogenesis: coordinating partners with multiple functions. Nat Rev Neurosci 2014;15;703-18.

2. Li L, Xiong WC, Me IL. Neuromuscular junction formation, aging, and disorders. Ann Rev Physio 2018;80:159-88.

3. Murray LM, Comley LH, Thomson D, Parkinson N, Talbot K, Gillingwater TH. Selective vulnerability of motor neurons and dissociation of pre-and post-synaptic pathology at the neuromuscular junction in mouse models of spinal muscular atrophy. Hum Mol Genet 2008;17:949-62.

4. Shen, Lu Y, Zhang B, Figueiredo D, Bean J, Jung J, et al. Antibodies against low-density lipoprotein receptor-related protein 4 induce myasthenia gravis. $\mathrm{J}$ Clin Invest 2013;123:5190-202.

5. Krause Neto W, Silva W, Ciena AP, Bocalini D, Anaruma C, Gama E. Divergent effects of resistance training and anabolic steroid on the postsynaptic region of different skeletal muscles of aged rats. Exp Gerontol 2017;80-90.

6. Tu WY, Xu W, Zhang K, Shen C. Whole-mount staining of neuromuscular junctions in adult mouse diaphragms with a sandwich-like apparatus. J Neurosci Meth 2020;350:109016.

7. Rocha LC, Jacob C dos S, Barbosa GK, Pimentel Neto JP, Krause Neto W, Gama EF, et al. Remodeling of the skeletal muscle and postsynaptic component after short-term joint immobilization and aquatic training. Histochem Cell Biol 2020;154:621-8.

8. Tajsharghi, H. Thick and thin filament gene mutations in striated muscle diseases. Int J Mol Sci 2008;9:1259-75.

9. $\mathrm{Wu} \mathrm{H}$, Xiong WC, Mei L. To build a synapse: signaling pathways in neuromuscular junction assembly. Development 2010;137:1017-33.

10. Farenia R, Lesmana R, Uchida K, Iwasaki T, Koibuchi N, Shimokawa N. Changes in biomarker levels and myofiber constitution in rat soleus muscle at different exercise intensities. Mol Cell Biochem 2019;458:9-87.

11. Wilson MH, Deschenes MR. The neuromuscular junction: anatomical features and adaptations to various forms of increased or decreased neuromuscular activity. Int J Neurosci 2005;115:803-28.

12. Lovering RM, Iyer SR, Edwards B, Davies KE. Alterations of neuromuscular junctions in Duchenne muscular dystrophy. Neurosci Lett 2020;737:135304.

13. Gonzalez-Freire M, Semba RD, Ubaida-Mohein C, Fabbri E, Scalzo P, Hojlund K, et al. The human skeletal muscle proteome project: a reappraisal of the current literature. J Cachexia Sarcopenia Muscle 2016;8:5-18.

14. Deschenes MR, Hurst TE, Ramser AE, Sherman EG. Presynaptic to postsynaptic relationships of the neuromuscular junction are held constant across age and muscle fiber type. Dev Neurobiol 2013;73:744-53.

15. Deschenes MR, Sherman EG, Roby MA, Glass EK, Harris MB. Effect of resistance training on neuromuscular junctions of young and aged muscles featuring different recruitment patterns. J Neurosci Resh 2015;93:504-13.

16. Deschenes MR, Tufts HL, LI S, Noronha AL, Adan MA.
Effects of exercise training on neuromuscular junctions and their active zones in young and aged muscles. Neurobiol Aging 2020;95:1-8.

17. Krause Neto W, Silva W, Ciena AP, Bocalini D, Nucci R, et al. Total training load may explain similar strength gains and muscle hypertrophy seen in aged rats submitted to resistance training and anabolic steroids. Aging Male 2018;21:65-76.

18. Rocha LC, Pimentel Neto JP, Sant'Ana JS, Jacob C dos S, Barbosa GK, Krause Neto W, et al. Repercussions on sarcomeres of the myotendinous junction and the myofibrillar type adaptations in response to different trainings on vertical ladder. Microsc Res Techn 2020;83:1190-7.

19. Krause Neto W, Silva WA, Ciena AP, Nucci RAB, Anaruma CA, Gama EF. Effects of strength training and anabolic steroid in the peripheral nerve and skeletal muscle morphology of aged rats. Front Aging Neurosci 2017;9:205.

20. Pimentel Neto J, Rocha LC, Klein G.B, Jacob C dos S, Krause Neto W, Watanabe I, et al. Myotendinous junction adaptations to ladder-based resistance training: identification of a new telocyte niche. Sci Rep 2020;10:14124.

21. Krause Neto WN, Silva W, Ciena AP, Bocalini D, Anaruma C, Gama E. Divergent effects of resistance training and anabolic steroid on the postsynaptic region of different skeletal muscles of aged rats. Exp Gerontol 2017;98:80-90.

22. Lourenço I, Krause Neto W, Amorim LSP, Ortiz VMM, Geraldo VL, Ferreira GHS, et al. Muscle hypertrophy and ladder-based resistance training for rodents: A systematic review and meta-analysis. Physio Rep 2020;8:14502.

23. Hornberger TAJ, Farrar RP. Physiological hypertrophy of the FHL muscle following 8 weeks of progressive resistance exercise in the rat. Can J Appl Physiol 2004;29:16-31.

24. Jones RA, Reich CD, Dissanayake KN, Kristmundsdottir F, Findlater GS, Ribchester RR, et al. NMJ-morph reveals principal components of synaptic morphology influencing structure - Function relationships at the neuromuscular junction. Open Biol 2017;6:160240.

25. Fuentes I, Cobos AR, Segade LAG. Muscle fibre types and their distribution in the biceps and triceps brachii of the rat and rabbit. J Anat 1998;192:203-10.

26. Gundersen K. Excitation-transcription coupling in skeletal muscle: the molecular pathways of exercise. Biol Rev 2011;86:564-600.

27. Talbot J, Maves L. Skeletal muscle fiber type: using insights from muscle developmental biology to dissect targets for susceptibility and resistance to muscle disease. Wiley Interdiscip Rev Dev Biol 2016;5:518-34.

28. Qaisar R, Bhaskaran S, Van Remmen H. Muscle fiber type diversification during exercise and regeneration. Free Radic Biol Med 2016;98:56-67.

29. Pette D. Training effects on the contractile apparatus. Acta Physiol Scand 1998;162:367-76.

30. Schiaffino S, Reggiani C. Fiber types in mammalian skeletal muscles. Physiol Rev 2011;91:1447-31.

31. Jorgensen KW, Phillips SM, Hornberger TA. Identifying the structural adaptations that drive the mechanical load-induced growth of skeletal muscle: A scoping review. Cells 2020;9:1658.

32. Esbjörnsson M, Hellsten-Westing Y, Balsom PD, Sjödin B, Jansson E. Muscle fibre type changes with sprint training: effect of training pattern. Acta Physiol Scand 1993;149:245-6.

33. Chen J, Mizushige T, Nishimune H. Active zone density is conserved during synaptic growth but impaired in aged mice. J Comp Neurol 2012;520:434-52.

34. Neher E. Vesicle pools and Ca21 microdomains: Review new tools for understanding their roles in neurotransmitter release. 
Neuron 1998;20:389-99.

35. Deschenes MR, Roby MA, Glass EK. Aging influences adaptations of the neuromuscular junction to endurance training. Neuroscience 2011;190:56-66.

36. Arnold AS, Gill J, Christe M, Ruiz R, Mcguirk S, St-Pierre J, et al. Morphological and functional remodelling of the neuromuscular junction by skeletal muscle PGC-1 $\alpha$. Nat Comm 2014;5:3569.

37. Tintignac LA, Brenner HR, Rüegg MA. Mechanisms regulating neuromuscular junction development and function and causes of muscle wasting. Physiol Rev 2015;95:809-52.

38. Jianjun MA, Smith BP, Smith TL, Walker FO, Rosencrance EV, Koman LA. Juvenile and adult rat neuromuscular junctions: density, distribution, and morphology. Muscle Nerve 2002;26:804-9.

39. Häkkinen K, Alen M, Kraemer WJ, Gorostiaga E, Izquierdo
M, Rusko H, et al. Neuromuscular adaptations during concurrent strength and endurance training versus strength training. Eur J Appl Physiol 2003;89:42-52.

40. Fyfe JJ, Bishop DJ, Stepto NK. Interference between concurrent resistance and endurance exercise: molecular bases and the role of individual training variables. Sports Med 2014;44:743-62.

41. Lourenco MV, Frozza RL, de Freitas GB. Exercise-linked FNDC5/irisin rescues synaptic plasticity and memory defects in Alzheimer's models. Nat Med 2019;25:165-75.

42. Feng YS, Yang SD, Tan ZX, Wang MM, Xing Y, Dong F, et al. The benefits and mechanisms of exercise training for Parkinson's disease. Life Sci 2020;245:117345.

43. Pimentel J, Tojal R, Morgado J. Epilepsy and physical exercise. Seizure 2015;25:87-94.

Received for publication: 15 May 2021. Accepted for publication: 14 July 2021.

This work is licensed under a Creative Commons Attribution-NonCommercial 4.0 International License (CC BY-NC 4.0).

CCopyright: the Author(s), 2021

Licensee PAGEPress, Italy

European Journal of Histochemistry 2021; 65(s1):3274

doi:10.4081/ejh.2021.3274 